\title{
Metaplastic Carcinoma with Mesenchymal Differentiation in Augmented Breast using Liquid Silicone Injection: A Case Report
}

Lara Mae Lorenzo, Sarah Jane Datay-Lim, Jose Carnate Jr.

Department of Laboratory Medicine and Pathology, The Medical City, Pasig City, Philippines

\section{ABSTRACT}

The relationship between the use of liquid silicone for breast augmentation and carcinogenesis remains undetermined due to limited data reported, especially regarding its risks for acquiring cancer. We documented a case of an 81-year-old woman who presented with bilateral enlarging breast masses with a known history of breast augmentation using liquid silicone. On microscopic examination, the malignancy showed both mesenchymal and epithelial components in a background of stromal changes related to liquid silicone. Based from morphology and immunohistochemistry studies (P63, CK, HMW-CK, and $C K 5 / 6, C D 34$, and BCL-2), this case was signed out as metaplastic carcinoma with mesenchymal differentiation. This rare case of metaplastic carcinoma with mesenchymal differentiation coexisting with liquid silicone, provides evidence supporting the link between cancer development and siliconomas.

Key words: metaplastic breast carcinoma, liquid silicone, breast augmentation, invasive breast carcinoma

ISSN 2507-8364 (Online)

Printed in the Philippines. Copyright $\left({ }^{\circ} 2020\right.$ by the PJP.

Received: 23 October 2020

Accepted: 29 November 2020.

Published online first: 16 December 2020.

https://doi.org/10.21141/PJP.2020.10

Corresponding author: Lara Mae S. Lorenzo, RMT, MLS $\left(A S C P^{i}\right)^{C M}, M D$

E-mail:yamsylorenzo@gmail.com

\section{INTRODUCTION}

The practice of using liquid silicone for breast augmentation was popular between 1944 to the early $1990 \mathrm{~s}^{1,2}$ but was eventually banned by the USA Food and Drug Administration. This was due to findings of breast complications such as inflammatory changes and fibrosis in patients who had liquid silicone breast augmentation. ${ }^{1}$

To this date, liquid silicone injection for breast augmentation is still performed illegally in the Philippines by unlicensed and unskilled practitioners. A fiveyear retrospective study was able to report the benign complications of foreign body injection to the breast. $^{3}$ Yet, no literature was published and reported locally (HERDIN Plus and Philippine E-Journals) regarding the coexistence of silicone mastopathy and a malignant breast neoplasm, specifically with a metaplastic carcinoma.

This paper reports the finding of breast malignancy in a patient with breast augmentation. Microscopically, the tumor was adjacent to stromal changes associated with liquid silicone.

\section{CASE}

This is a case of an 81-year old woman who presented with bilateral enlarging breast masses, 5 years prior to consultation. Patient had a 30-year history of using liquid silicone injection as augmentation. On physical examination, the palpable masses were both firm, movable and irregular. The left breast mass was noted to be more tender, larger and heavier in size. Patient underwent excision of the masses and the specimens were sent for histopathologic evaluation. No radiological examination was done prior to excision biopsy.

On gross examination, two pink-tan ovoid doughy tissues were received and with the following measurements: "Right breast mass" - 240 grams and 11.2 x 8.5 x $3.2 \mathrm{~cm}$; 


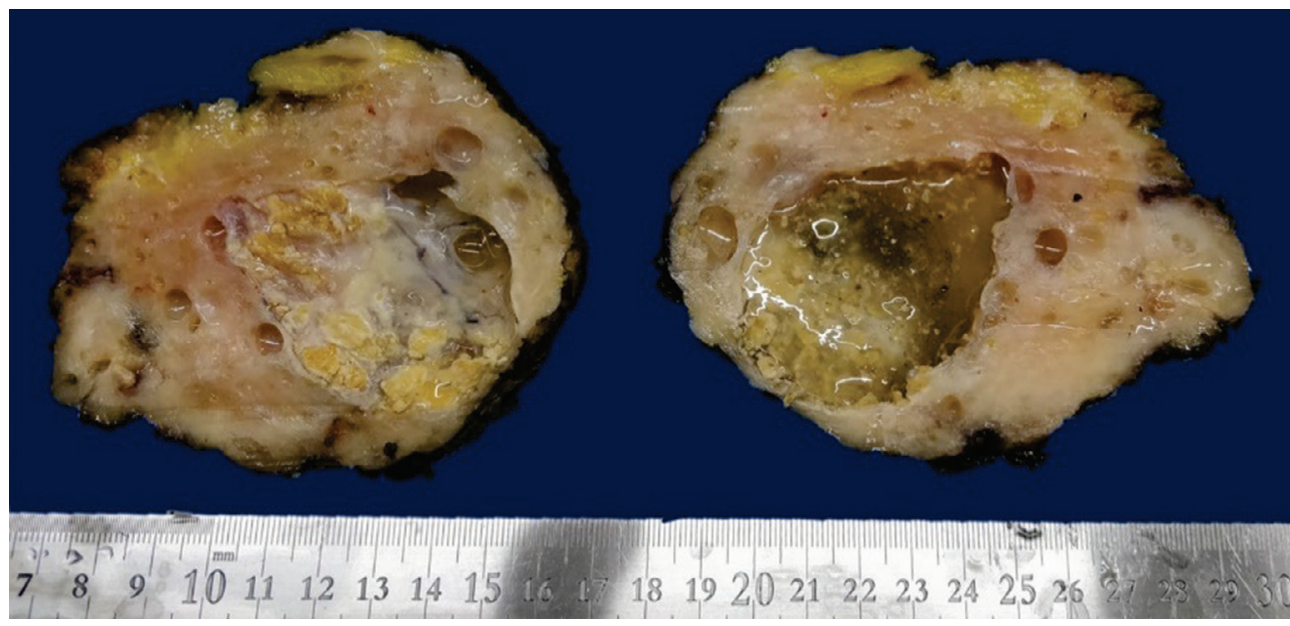

Figure 1. Gross appearance of the excision biopsy specimen of the right breast mass. Cut section of the mass show multiple cystic spaces filled with clear viscous material, largest cyst having multiple yellow-tan gritty tissue fragments.

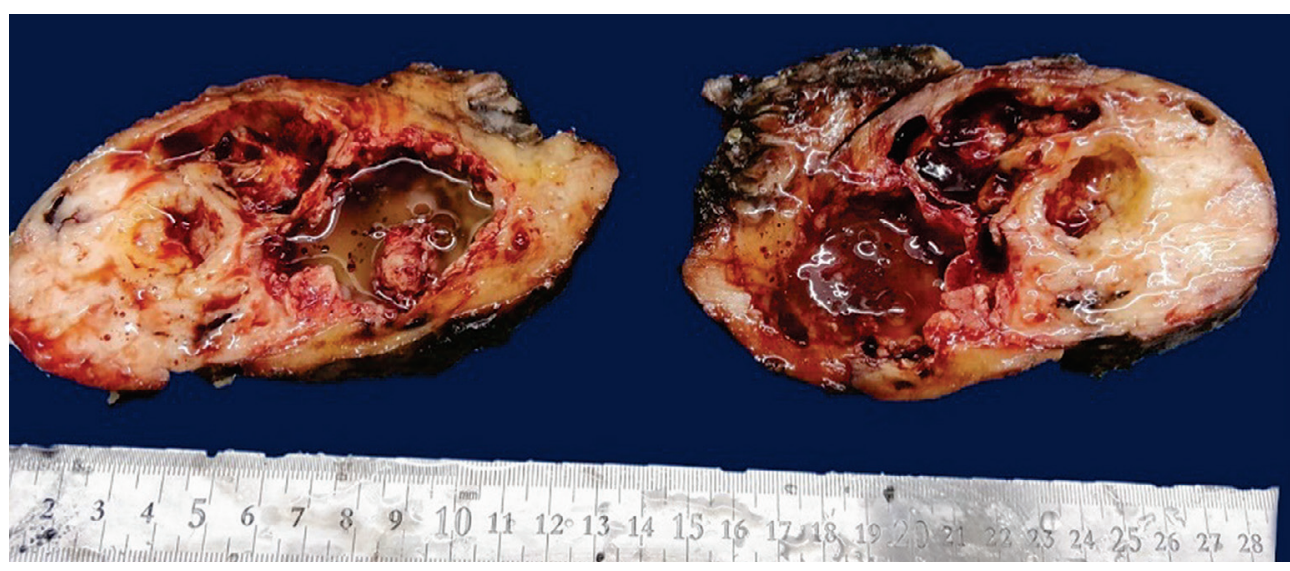

Figure 2. Gross appearance of the excision biopsy specimen of the left breast mass. Cut sections show a cream to red-tan variegated and gritty cut surface, with multiple cystic cavities filled with clear viscous material and red-brown clot-like material. The inner lining of the largest cavity was nodular.

"Left breast mass" -580 grams and 13.4 x $9.5 \times 7.1 \mathrm{~cm}$. Cut sections of the right breast mass showed multiple cystic cavities, measuring up to $6.0 \mathrm{~cm}$ in widest dimensions, filled with clear viscous material (Figure 1). Cut sections of the left breast mass showed multiple cystic cavities which measure up to $4.5 \mathrm{~cm}$ in widest dimensions, filled with clear viscous material and red-brown clot-like material (Figure 2). The inner lining of the largest cavity is nodular with a tan-white gritty cut surface. The rest of the left breast mass had a cream to red-tan variegated and gritty cut surface.

Histopathologic examination of the right breast showed foreign-body changes with numerous cystic spaces and vacuoles, consistent with silicone mastopathy (Figure 3).

However, microscopic examination of the left breast mass showed a malignancy composed of tumor cells surrounded by stroma with varying degrees of ossification and chondromyxoid changes (Figures 4 to 5). Tumor cells were noted to have enlarged, markedly pleomorphic and hyperchromatic nuclei, prominent nucleoli, coarse

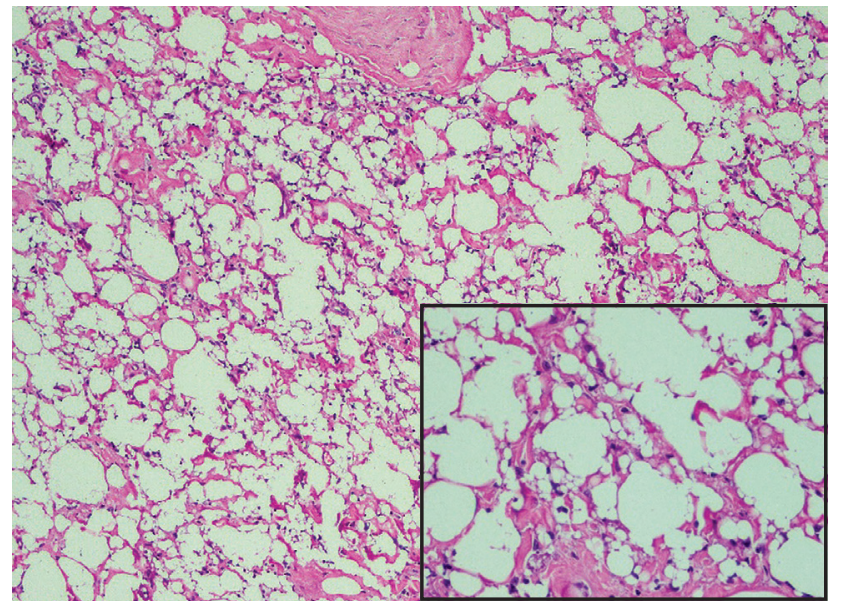

Figure 3. Extensive involvement of silicone to the breast tissue (right). The background is composed of chronic inflammation and reactive fibrosis. Silicone has been leached from the fixation processed and is visualized as empty vacuoles or refractile colorless material on closer magnification (Hematoxylin-Eosin, 100x, Inset 400x). 
chromatin pattern and ample eosinophilic cytoplasm (Figure 6). Adjacent to the carcinoma are findings of foreign-body changes with numerous cystic spaces and vacuoles, consistent also with silicone mastopathy (Figure 4).

Immunohistochemistry studies were done and showed that the tumor cells stained weakly and focally positive for p63, CK, HMW-CK, and CK5/6, and stained negative for CD34 and BcL-2 (Figure 7). For the hormone receptor assay, ER, PR and HER-2/neu were negative (Figure 7). Given the morphologic and immunohistochemical profile of the mass, this case was signed out as metaplastic carcinoma with mesenchymal differentiation (syn. Matrixproducing metaplastic carcinoma).

\section{DISCUSSION}

Metaplastic breast carcinomas with mesenchymal elements subtype, formerly called as matrix-producing metaplastic carcinoma, are invasive carcinoma with direct transition to osseous and/or cartilaginous matrix, with or without an intervening spindle cell component., $, 5,6$ Considered as extremely rare and aggressive ${ }^{4,7,8}$, the mean age of patients with this malignancy is 56 years. $^{9}$ Clinically and mammographically, presentation is similar to invasive ductal carcinoma. ${ }^{8}$ Microscopically, they are mainly composed of two kinds of populations: the mesenchymal and epithelial. The mesenchymal elements would often include chondroid, osseous, rhabdomyoid and even neuroglial differentiations. The epithelial areas would be present in the forms of glandular differentiation, in tubules or in solid clusters, and/or foci of squamous differentiation. ${ }^{6}$

Differential diagnoses for a metaplastic carcinoma with a mesenchymal differentiation would include the following: high-grade phyllodes tumor, primary breast sarcoma, extraskeletal osteosarcoma, and myoepithelial carcinoma. Just like in our case, identification of overt epithelial component would be difficult especially if most tumor cells have already undergone metaplastic changes. Hence, immunohistochemical studies are helpful, wherein the epithelial component would stain positive for cytokeratin (CK), while the mesenchymal / matrixproducing component will stain negative for CK but positive for $\mathrm{S} 100 .{ }^{8}$

For this carcinoma (or even any malignancy), diagnostic dilemmas arise with a concomitant foreign body injectable material or even the use of implants. On mammography, prior use of liquid silicone, silicone gel-filled and salinefilled implants, can hinder the accurate delineation between a silicone granuloma and breast carcinoma. The opaque densities (similar to fat) would obscure the malignancy, ultimately hindering the early diagnosis of a coexisting neoplasm. ${ }^{10,11}$ Augmented patients would have a higher false-negative mammography rate compared to nonaugmented patients. Despite the augmented group of patients showing a slightly greater risk of invasive tumors, higher frequency of palpable mass and a higher incidence rate of metastasis to the axillary lymph nodes, both groups showed no statistically difference in terms of disease stage, tumor size, recurrence rates, and survival

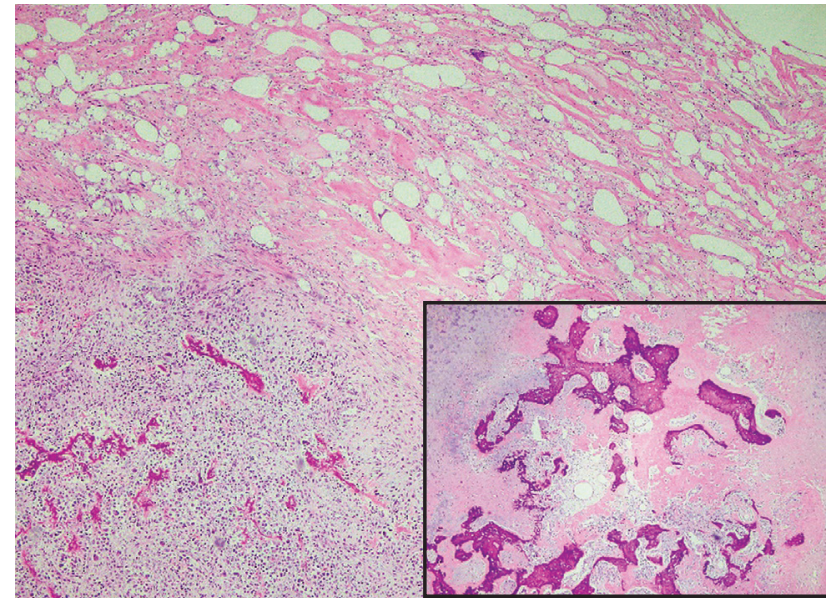

Figure 4. Metaplastic carcinoma with mesenchymal differentiation with adjacent silicone mastopathy of the left breast. On closer magnification, it is composed of an overt carcinoma with direct transition to an osseous stromal matrix (Hematoxylin-eosin, 40x, Inset 100x).

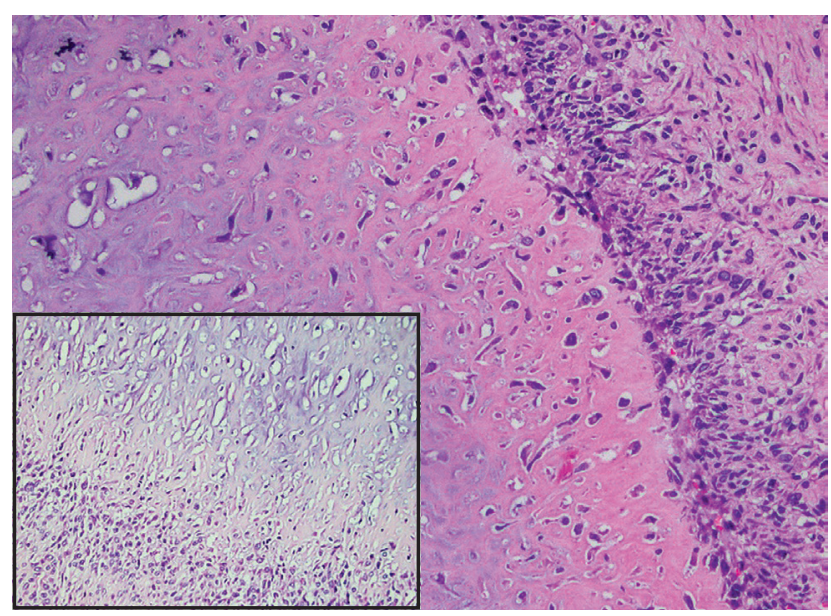

Figure 5. Matrix-producing metaplastic carcinoma with poorly differentiated carcinomatous cells at the periphery of a chondromyxoid stroma, left breast (Hematoxylin-eosin, 200x, Inset 200x).

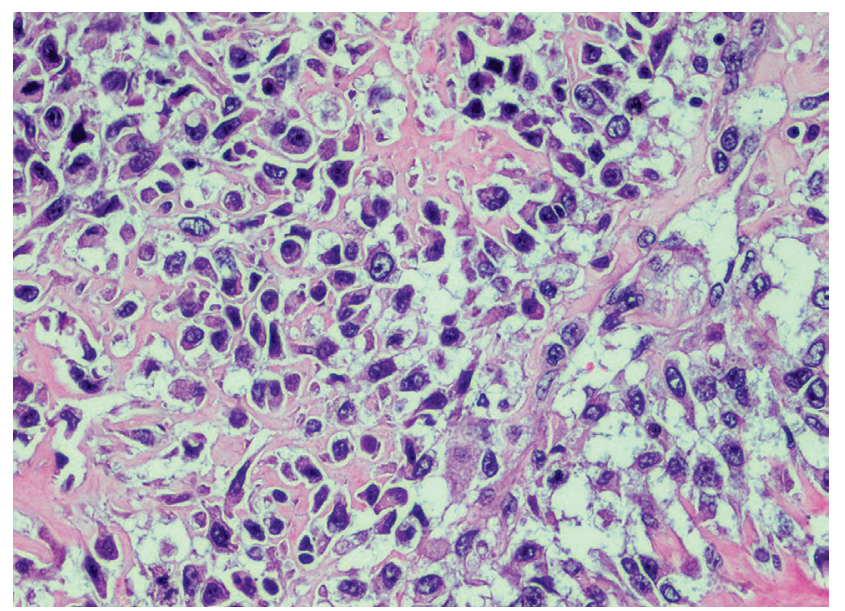

Figure 6. Tumor cells exhibiting enlarged, markedly pleomorphic and hyperchromatic nuclei, prominent nucleoli, coarse and vesicular chromatin patterns and ample eosinophilic cytoplasm (Hematoxylin-eosin, 100x). 

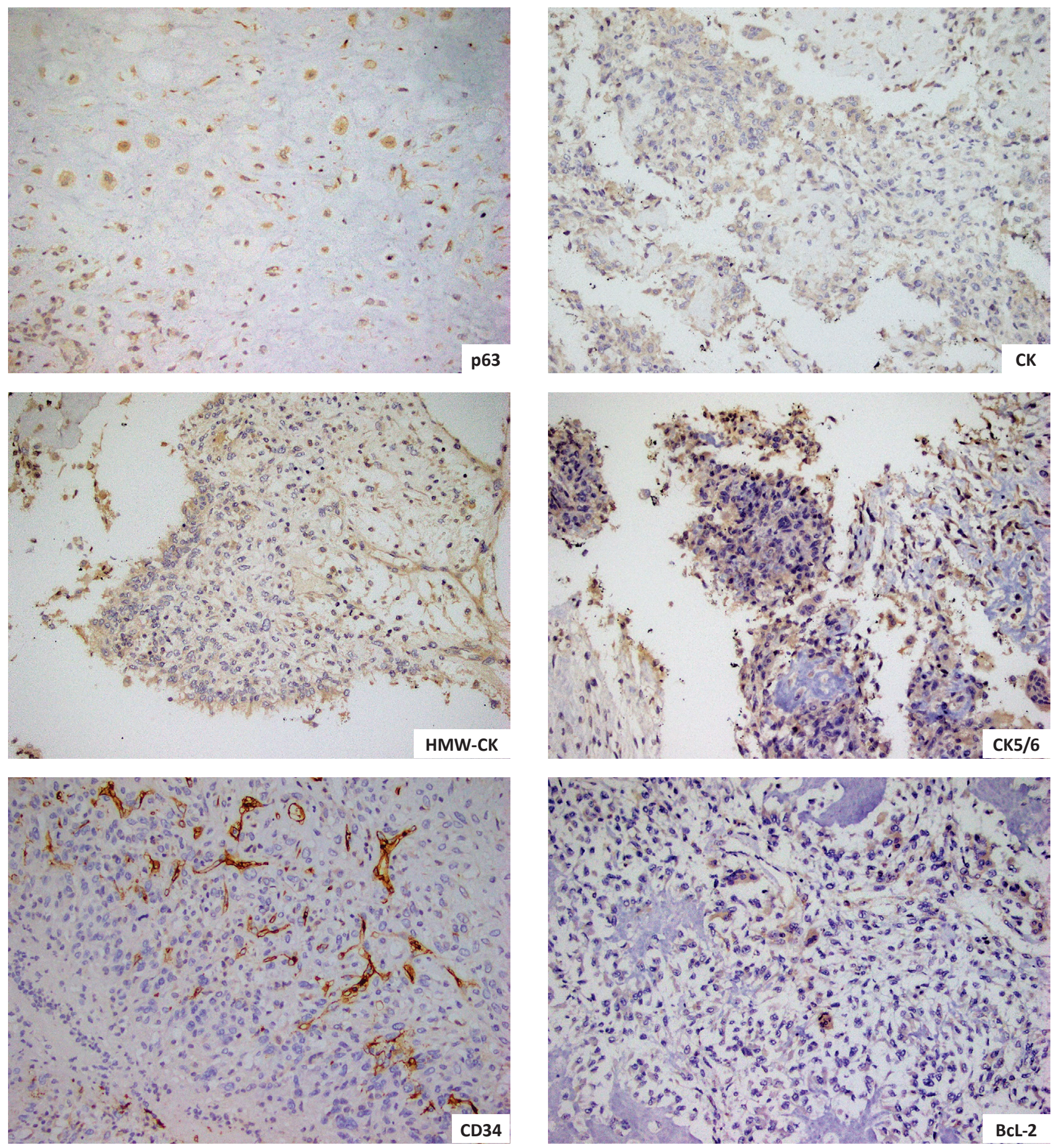

Figure 7. Immunohistochemistry showing: focal and weak nuclear positivity staining for p63; focal and weak cytoplasmic positivity staining for $\mathrm{CK}, \mathrm{HMW}-\mathrm{CK}$ and $\mathrm{CK} 5 / 6$; negative staining for CD34 and BCL-2, and a triple negative receptor (ER, PR, HER-2/neu) assay (see next page) (Horse radish peroxidase method, 100x).

rate. One explanation would be that the presence of implants would help facilitate the palpation of the tumor, thus are easier to clinically examine compared to the nonaugmented. ${ }^{10}$

On the histopathologic context, liquid silicone appears as empty vacuoles or spaces, which has been washed out during tissue preparation. Foreign body giant cells, vascular olibiterans, stromal fibrosis, and chronic inflammation would also be appreciated. ${ }^{1}$ For its causal relationship with carcinogenesis, there is still no definite consensus regarding it. ${ }^{2,12}$ According to a study done by Stivala et al., major cohort studies were done prior which demonstrated a $0.2 \%$ to $2.7 \%$ breast cancer frequency rate following augmentation. ${ }^{13}$ Although many cases of breast cancer with prior augmentation have been reported, ${ }^{3,10,13}$ data specific to liquid silicone is still limited. . $^{211,14}$ Most studies were attributed from using silicone gel implants. ${ }^{10}$ However, Morgenstern et al were able to discuss their findings of 12 patients with free silicone and breast cancer. 

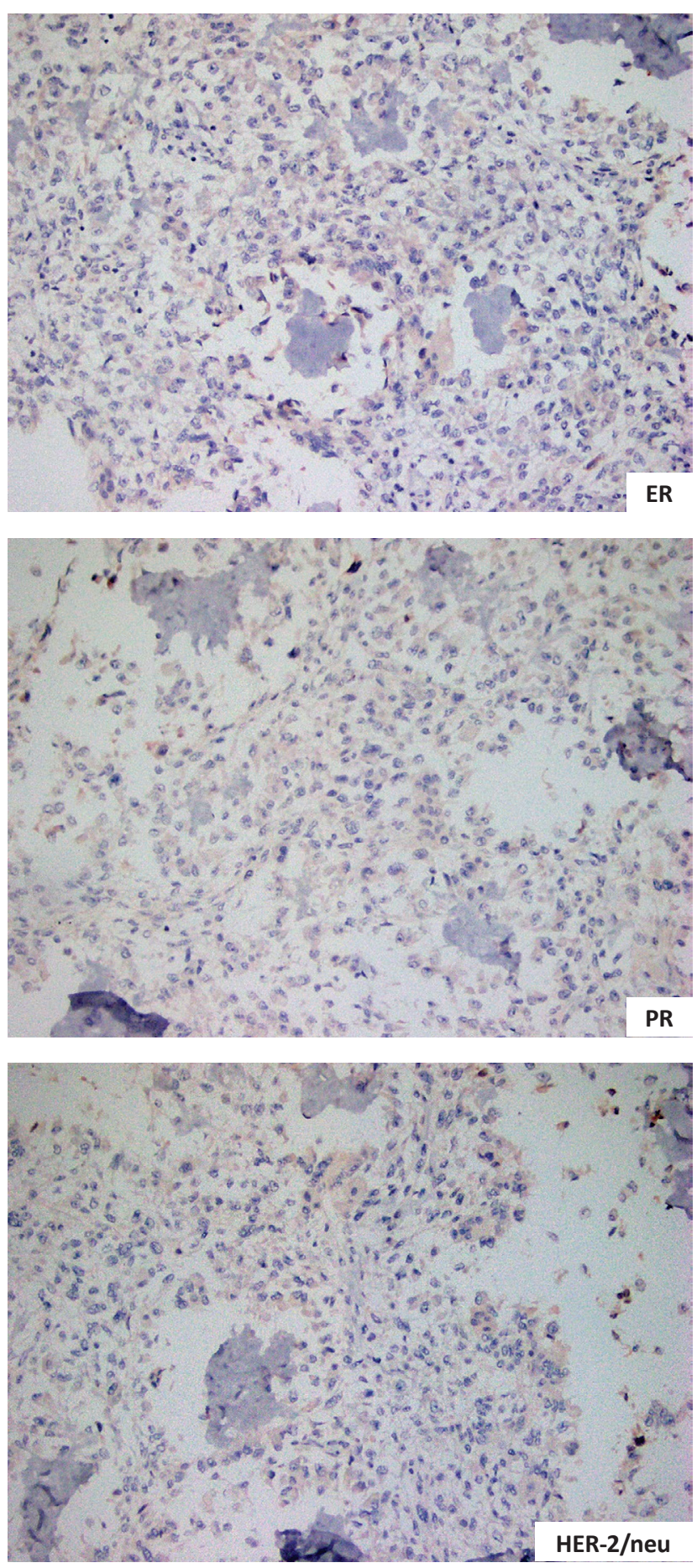

Their study postulated that free silicone may provide a more attractive medium for tumor cells to proliferate. Other pathologic findings were the lack of desmoplastic reaction of the tumor when admixed with silicone and tumors showing usual desmoplastic reaction showed little fibrosis in areas away from silicone. A study done by Tanaka et al reported that the malignant cells of the tumors diagnosed as invasive micropapillary carcinoma had close contact with the liquid silicone, which they considered as an evidence as a link between carcinogenesis and use of silicone. ${ }^{12}$ And to support the higher incidence of metastasis to the axillary lymph nodes, the pathologic findings of the same study suggested that free silicone causes the following: an abnormal opening of the lymphatic channels, facilitating the spread of tumor cells; the inhibition of tumor-induced desmoplasia; or from an altered immune mechanism associated with silicone granuloma. ${ }^{2}$ Despite these observations and evidences reported, data was still considered limited by the authors due to lack of thorough investigation between breast cancer and liquid silicone. ${ }^{3,12,14}$

Metaplastic breast carcinomas with mesenchymal elements subtype are hormone receptor negative (ER, PR, and HER-2), and radiotherapy and chemotherapy are of limited effectiveness. No standard treatment has been established yet. Recent clinical trials have shown targeted gene therapy plays a role following genetic profiling. ${ }^{15}$ But for silicone mastopathy with coexistence of this carcinoma (and even this carcinoma alone), surgery is still considered the treatment of choice. ${ }^{2,14}$

\section{CONCLUSION}

Metaplastic carcinoma with mesenchymal elements is considered by most publications as rare. To the authors present knowledge, there have been no local reported cases of having this malignancy after breast augmentation with liquid silicone. Although there is no established relationship between liquid silicone and carcinoma, our findings suggest a link between the two.

\section{ETHICAL CONSIDERATION}

All attempts were done to acquire a consent from the patient, however, was lost to follow-up. This case report was made in accordance to the principles based on the Declaration of Helsinki.

\section{STATEMENT OF AUTHORSHIP}

All authors certified fulfillment of ICMJE authorship criteria.

\section{AUTHOR DISCLOSURE}

The authors declared no conflict of interest.

\section{FUNDING SOURCE}

\section{None.}

\section{REFERENCES}

1. Peters W, Fornasier V. Complications from injectable materials used for breast augmentation. Can J Plast Surg. 2009;17(3):89-96. PMID: 20808751. PMCID: PMC2740603. https://doi.org/ $10.1177 / 229255030901700305$

2. Morgenstern L, Gleischman S, Michel SL, Rosenberg JE, Knight I, Goodman D. Relation of free silicone to human breast carcinoma. Arch Surg. 1985;120(5): 573-7. PMID: 2985027. https://doi.org/10.1001/ archsurg. 1985.01390290051008

3. Baluyut-Angeles KVA, Arcilla EPE. A Five-year retrospective study of the demographic distribution, clinical profile and treatment of patients with foreign body injections to the breast seen by the division of 
plastic surgery at the Philippine General Hospital. PJSS. 2011; 66(2):60-7. https://pcs.org.ph/assets/ journals/PJSS-66-2-Apr-Jun-2011-4.pdf.

4. Bhosale SJ, Kshirsagar AY, Sulhyan SR, Sulhyan SR, Jagtap SV. Matrix-producing metaplastic breast carcinoma - a rare malignancy. Am J Case Rep. 2013;14:213-215. PMID: 23826471. PMCID: PMC3700454. https://doi.org/10.1x2659/ajcr.883958.

5. Koufopoulos N, Kokkali S, Antoniadou F, Dimas DT, Missitzis IL. Matrix-producing breast carcinoma: a rare subtype of metaplastic breast carcinoma. Cureus. 2019;11(7):e5188. PMID: 31565596. PMCID: PMC6758965. https://doi.org/10.7759/cureus.5188

6. WHO Classification of tumours Editorial Board. Breast tumours, 5th ed. Lyon France: International Agency for Research on Cancer; 2019. https://publications. iarc.fr $/ 581$.

7. Rossi L, Paglicci C, Caprio G, et al. Matrix-producing carcinoma of the breast: a case report. Case Rep Oncol. 2013;6(2):245-9. PMID: 23741218. PMCID: PMC3670645. https://doi.org/10.1159/000351119

8. Shruti S, Siraj F. Matrix-producing metaplastic breast carcinoma - a rare tumor with heterologous elements. Ger Med Sci. 2017;15:Doc17. PMID: 29255401. PMCID: PMC5727344. https://doi.org/ $10.3205 / 000258$.

9. Rosen's breast pathology, 4th ed. Philadelphia, PA: Lippincott Williams \& Wilkins; 2014

10. Handel N, Silverstein MJ. Breast cancer diagnosis and prognosis in augmented women. Plast Reconstr Surg. 2006;118(3):587-93; discussion 594-6. PMID: 16932162. https://doi.org/10.1097/01.prs.0000233038.47009.04
11. Cheung YC, Lee KF, Ng SH, Chan SC, Wong AM. Sonographic features with histologic correlation in two cases of palpable breast cancer after breast augmentation by liquid silicone injection. J Clin Ultrasound. 2002;30(9): 548-51. PMID: 12404521. https://doi.org/10.1002/jcu.10110.

12. Tanaka Y, Morishima I, Kikuchi K. Invasive micropapillary carcinomas arising 42 years after augmentation mammoplasty: a case report and literature review. World J Surg Oncol. 2008;6:33. PMID: 18341700. PMCID: PMC2292720. https://doi. org/10.1186/1477-7819-6-33.

13. Stivala A, Libra M, Stivala F, Perrotta R. Breast cancer risk in women treated with augmentation mammoplasty (review). Oncol Rep. 2012; 28(1): 3-7. PMID: 22576794. https://doi.org/10.3892/ or.2012.1762.

14. Nakahori R, Takahashi R, Akashi M, et al. Breast carcinoma originating from a silicone granuloma: a case report. World J Surg Oncol. 2015;13:72. PMID: 25888835. PMCID: PMC4350903. https://doi. org/10.1186/s12957-015-0509-6

15. McMullen ER, Zoumberos NA, Kleer CG. Metaplastic breast carcinoma: update on histopathology and molecular alterations. Arch Pathol Lab Med. 2019;143(12):1492-6. PMID: 31765246. https://doi. org/10.5858/arpa.2019-0396-RA.

\footnotetext{
Disclaimer: This journal is OPEN ACCESS, providing immediate access to its content on the principle that making research freely available to the public supports a greater global exchange of knowledge. As a requirement for submission to the PJP, all authors have accomplished an AUTHOR FORM, which declares that the ICMJE criteria for authorship have been met by each author listed, that the article represents original material, has not been published, accepted for publication in other journals, or concurrently submitted to other journals, and that all funding and conflicts of interest have been declared. Consent forms have been secured for the publication of information about patients or cases; otherwise, authors have declared that all means have been exhausted for securing consent.
} 